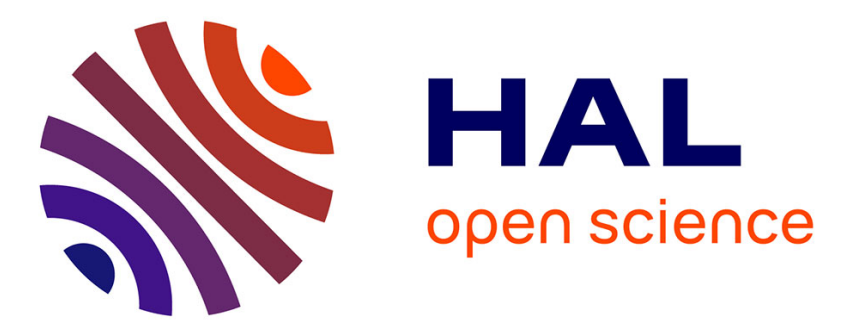

\title{
Fast and Accurate Segmentation Method of Active Shape Model with Rayleigh Mixture Model Clustering for Prostate Ultrasound Images
}

Hui Bi, Yibo Jiang, Hui Tang, Guanyu Yang, Huazhong Shu, Jean-Louis Dillenseger

\section{To cite this version:}

Hui Bi, Yibo Jiang, Hui Tang, Guanyu Yang, Huazhong Shu, et al.. Fast and Accurate Segmentation Method of Active Shape Model with Rayleigh Mixture Model Clustering for Prostate Ultrasound Images. Computer Methods and Programs in Biomedicine, 2020, 184, pp.105097. 10.1016/j.cmpb.2019.105097 . hal-02316147

\section{HAL Id: hal-02316147 \\ https://hal.science/hal-02316147}

Submitted on 15 Oct 2019

HAL is a multi-disciplinary open access archive for the deposit and dissemination of scientific research documents, whether they are published or not. The documents may come from teaching and research institutions in France or abroad, or from public or private research centers.
L'archive ouverte pluridisciplinaire HAL, est destinée au dépôt et à la diffusion de documents scientifiques de niveau recherche, publiés ou non, émanant des établissements d'enseignement et de recherche français ou étrangers, des laboratoires publics ou privés. 


\title{
Fast and Accurate Segmentation Method of Active Shape Model with Rayleigh Mixture Model Clustering for Prostate Ultrasound Images
}

\author{
Hui $\mathrm{Bi}^{1}$, Yibo Jiang ${ }^{2}$, Hui Tang ${ }^{3}$, Guanyu Yang ${ }^{3}$, Huazhong $\mathrm{Shu}^{3,4}$, \\ and Jean-Louis Dillenseger ${ }^{4,5}$
}

From:

${ }^{1}$ Changzhou University, Changzhou, China

${ }^{2}$ Changzhou Institute of Technology, Changzhou, China

${ }^{3}$ Laboratory of Image Science and Technology, School of Computer Science and Engineering, Southeast University, Nanjing, China

${ }^{4}$ Centre de Recherche en Information Biomédicale sino-français (CRIBs), Nanjing, China

${ }^{5}$ Univ Rennes, Inserm, LTSI - UMR 1099, F-35000 Rennes, France

* Corresponding author: shu.list@seu.edu.cn

\begin{abstract}
Background and Objective: The prostate cancer interventions, which need an accurate prostate segmentation, are performed under ultrasound imaging guidance. However, prostate ultrasound segmentation is facing two challenges. The first is the low signal-to-noise ratio and inhomogeneity of the ultrasound image. The second is the non-standardized shape and size of the prostate.

Methods: For prostate ultrasound image segmentation, this paper proposed an accurate and efficient method of Active Shape Model (ASM) with Rayleigh Mixture Model Clustering (ASM-RMMC). Firstly, Rayleigh Mixture Model (RMM) is adopted for clustering the image regions which present similar speckle distributions. These content-based clustered images are then used to initialize and guide the deformation of an ASM model.

Results: The performance of the proposed method is assessed on 30 prostate ultrasound images using four metrics as Mean Average Distance (MAD), Dice Similarity Coefficient (DSC), False Positive Error (FPE) and False Negative Error (FNE). The proposed ASM-RMMC reaches high segmentation accuracy with $95 \pm 1 \%$ for DSC, $1.87 \pm 0.51$ pixels for MAD, $2.10 \% \pm 0.36 \%$ for FPE and $2.78 \% \pm 0.7 \%$ for FNE, respectively. Moreover,
\end{abstract}


the average segmentation time is less than $8 \mathrm{~s}$ when treating a single prostate ultrasound image through ASM-RMMC.

Conclusions: This paper presents a method for prostate ultrasound image segmentation, which achieves high accuracy with less computational complexity and meets the clinical requirements.

\section{Keywords}

Ultrasound image; Prostate ultrasound image segmentation; Active shape model; Rayleigh mixture model

\section{Nomenclature}

\begin{tabular}{ll}
\hline Variable & Denote \\
\hline $\mathbf{x}^{i}$ & Points set of each training image \\
$\overline{\mathbf{x}}$ & Mean shape \\
$\mathbf{S}$ & Covariance matrix \\
$\mathbf{P}$ & Eigenvector \\
$\mathbf{g}_{\mathbf{j}}$ & Gradient profile \\
$\mathbf{Z}$ & Pixel intensities of the image \\
$\Psi$ & Parameters set of RMM \\
$\pi_{j}$ & Weight of each component in RMM \\
$\sigma_{j}$ & Covariance of each component in RMM \\
$\gamma_{i j}$ & Posterior probability of RMM \\
$p\left(z_{i} \mid \Psi\right)$ & RMM model \\
$p\left(z_{i} \mid \sigma_{j}\right)$ & Rayleigh distribution \\
\hline
\end{tabular}

\section{Introduction}

Prostate cancer is the second-leading cause of deaths from cancer for American men [1]. In 2018, the American Cancer Society estimated that among these men diagnosed with prostate cancer, $82 \%$ would be alive and $18 \%$ would die after treatment [1]. Thanks to the minimal invasive interventions, treatment in the early stage often effective to save the patients' lives. There are several minimal invasive interventional procedures such as puncture, brachytherapy, and High Intensity Focused Ultrasound (HIFU) [2, 3, 4]. Puncture is a diagnostic but also a therapeutic technique in which the puncture needles are used to inject drugs into the body cavity [4]. Brachytherapy, also known as internal radiation therapy, is a form of radiation therapy, in which the sources of radiation are placed in or near the target [2]. HIFU 
is a technology that inserts an ultrasound probe into the target tumor region to burn tumor cells with the thermal effect of high intensity ultrasound [3]. As a non-invasive treatment, HIFU has several advantages such as rapid recovery and low recurrence rate. The accurate delineation of the prostate boundary is crucial for intraoperative navigation to help surgeons accurately insert the probe to the target tumor region. However, several defects of the ultrasound imaging (e.g. attenuation, speckle, signal dropout, low contrast, signal shadowing, etc.) challenge the precise prostate boundary delineation [5].

Several classes of methods have been proposed for ultrasound images segmentation [6] that focus on edge detector [7], texture operators [8], and morphological operators [9]. However, these methods have limited validations since they do not make use of any prior knowledge of ultrasound physics. Recent works have shown that prior information in ultrasound images such as intensity, shape or temporal models can be introduced for segmentation $[10,11]$. Especially for prostate segmentation, shape models bring essential information since the anatomical structure of a healthy prostate is more likely an ellipse-shape. Gong et al. [10] propose a deformable superellipse model that drives the shape evolution by an efficient and robust Bayesian segmentation algorithm. Shen et al. [11] use a statistical shape model that adopts normalized features to make prostate shape invariant to the probe rotation. The experimental results of the aforementioned works show that incorporating tissue shape information can improve segmentation accuracy.

Active Shape Model (ASM), proposed by Cootes et al., is an effective tool that utilizes a set of points to represent variant shapes. Considering its ability to describe shapes based on a mean position and variant modes, the ASM is applied effectively for medical image segmentation $[12,13,14,15,16]$. The statistical shape model shows its potential for ultrasound image segmentation $[17,18]$, but the segmentation results are not as good as expected due to some specificity of the ultrasound images.

Speckle particularity plays a crucial role in the ultrasound images analysis $[5,6,19]$. One tissue in the ultrasound image appears as a region with similar speckle distribution. The statistics on the speckle as texture features can be used to handle ultrasound images segmentation [19, 20]. The most common model for speckle formation, known as fully developed speckle, indicates that the pixels' intensity in B-mode ultrasound images follows a Rayleigh probability density function [20, 21]. An application example is given in [22], where the morphological properties of the arterial vessel on intravascular ultrasound images (IVUS) are modeled by means of a Rayleigh distribution. Following this approach, some works have been done to model the tissue echo-morphology by a combination of Rayleigh distributions. Rayleigh Mixture Model (RMM) have been successfully employed in other fields $[23,24,25]$. Up to our knowledge, this is the first 
time to adopt RMM as descriptive speckle features for prostate ultrasound images segmentation. The proposed model combines prostate shape information represented by ASM and ultrasound image properties represented by RMM to achieve better segmentation.

In this paper, for ultrasound image prostate segmentation we propose a segmentation method using Active Shape Model with Rayleigh Mixture Model Clustering (ASM-RMMC), by combining the prostate physical shape priors and ultrasound image speckle properties. This method reduces ultrasound images inhomogeneity and improves the efficiency to estimate the shape deformations. Through segmentation experiments on clinical ultrasound images, the proposed method demonstrated that it is fast and accurate enough to meet the requirement of surgery.

\section{Materials and Methods}

\subsection{Active Shape Model}

\subsubsection{Principle}

The ASM is a flexible model that uses a set of points to represent the boundaries of objects in the image $[13,26]$. In this model, a shape is described by a set consisting of $n$ points $\mathbf{x}=\left\{\left(x_{1}, y_{1}\right),\left(x_{2}, y_{2}\right), \cdots,\left(x_{n}, y_{n}\right)\right\}$, as shown in Fig. 1. ASM also includes some gray level appearance around these points. The main idea is that a statistical model of the shape and the gray level appearance are created during the training phase based on manually labeled images. Then, the testing phase is applied to face with unlabeled images.

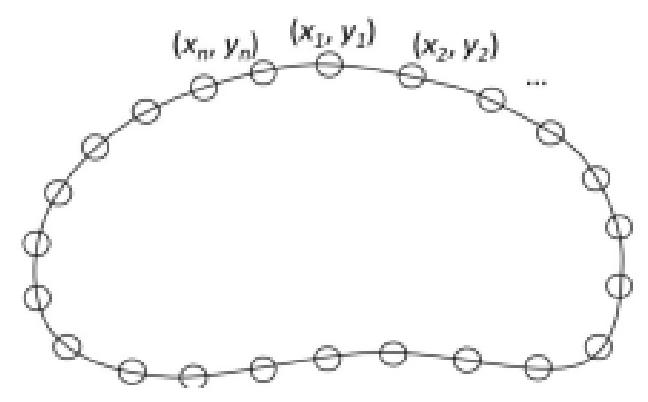

Figure 1: The description of a shape by a set consisting of $n$ points $\mathbf{x}=$ $\left\{\left(x_{1}, y_{1}\right),\left(x_{2}, y_{2}\right), \cdots,\left(x_{n}, y_{n}\right)\right\}$.

Training phase: Suppose that the training set contains $m$ manually labeled images. We have a set of points $\mathbf{x}^{i}$ for each image $i, i \in\{1,2, \ldots, m\}$ to describe each object boundary. Therefore, the whole training set contains $m$ boundary points set $\left\{\mathbf{x}^{1}, \mathbf{x}^{2}, \cdots, \mathbf{x}^{m}\right\}$. First, all the boundary points of the training set are aligned together using the Procrustes Analysis [27]. 
After the alignment, the average position of these points set can then be estimated by:

$$
\overline{\mathbf{x}}=\sum_{i=1}^{m} \mathbf{x}^{i} / m
$$

where $\mathbf{x}^{\prime i}, i \in\{1,2, \ldots, m\}$ denotes the aligned boundary points set. This process is illustrated in Fig. 2. The $m$ samples of the training set are shown in Fig. 2(a) while the aligned shapes $\mathrm{x}^{i}$ and the average shape are shown in Fig. 2(b).
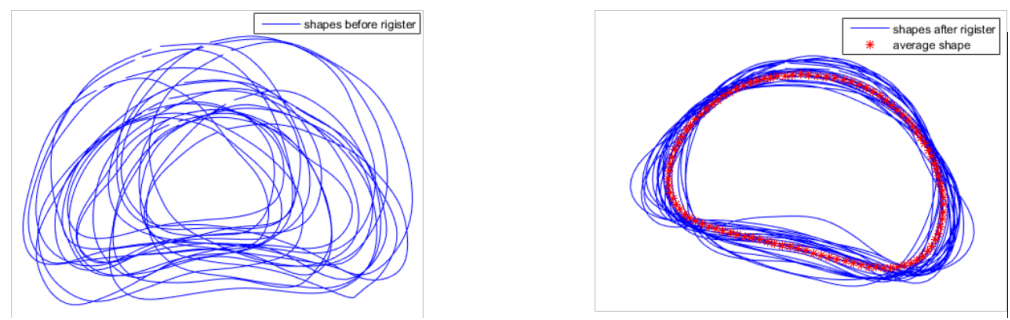

Figure 2: Illustration of manually delineated contours (a) and aligned contours with the average shape (in red) (b).

The covariance matrix $\mathbf{S}$ is defined as:

$$
\mathbf{S}=\sum_{i=1}^{m}\left(\mathbf{x}^{i}-\overline{\mathbf{x}}\right)^{T}\left(\mathbf{x}^{i}-\overline{\mathbf{x}}\right)
$$

The main variation modes can be obtained by applying the Principal Component Analysis (PCA). Let $\mathbf{P}$ be the first $t$ eigenvectors of $\mathbf{S}$. Therefore, any sample $\mathbf{x}^{i}$ in the training set, $i \in 1,2, \ldots, m$, can be described by $\overline{\mathbf{x}}, \mathbf{P}$ and $\mathbf{b}$ :

$$
\mathbf{x}^{i}=\overline{\mathbf{x}}+\mathbf{P b}, i \in 1,2, \ldots, m
$$

where $\mathbf{b}$ is a vector containing $t$ parameters.

Once the shape model is built, the statistics of the gray levels around the points should be included. As shown in Fig. $3, \mathbf{x}_{j}^{i}$ denotes the $j$ th point of the boundary points in the $i$ th sample. For the $j$ th point, a gray level profile is built by sampling the image along the boundary normal direction. This normal direction is approximated by the perpendicular of the line connecting the two neighbors of $\mathbf{x}_{j}^{i}$ : $\mathbf{x}_{j-1}^{i}$ and $\mathbf{x}_{j+1}^{i}$. We choose $k$ samples on each side of $\mathbf{x}_{j}^{i}$ and compute the derivative gray value to perform a gradient profile $\mathbf{g}_{j}^{i}$. Then, the normalized vector $\mathbf{g}_{j}$ composed by all the $m$ profiles is achieved by:

$$
\mathbf{g}_{j} \rightarrow \frac{1}{\sum_{i}\left|\mathbf{g}_{j}^{i}\right|} \mathbf{g}_{j}
$$




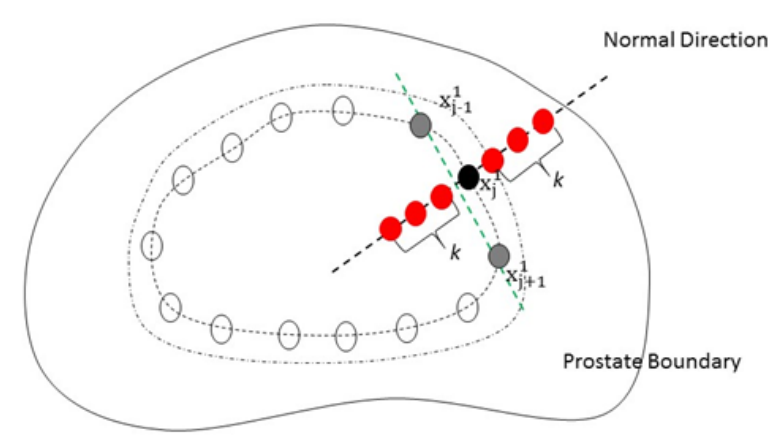

Figure 3: The gradient profile (shown in red dots) of the $j$ th boundary point (shown in black dot)

Testing phase. For unlabeled images, the idea is to deform the shape according to the mean shape $\overline{\mathbf{x}}$ and the statistics gray levels of each boundary point $\overline{\mathbf{x}}_{j}$. Firstly, the mean shape $\overline{\mathbf{x}}$ obtained in the training phase is used for initialization. From this initial pose, the model is matched using the following iterative approach. Then the statistics gray levels of each boundary point $\overline{\mathbf{x}}_{j}$ are considered:

1. In order to predict the point set $\mathbf{x}^{\text {new }}$ for the unlabeled image, the gray level appearance around the initial points $\overline{\mathbf{x}}_{j}, j \in 1,2, \ldots, n$, is examined in order to find the match points. The candidate points are sampled along the boundary normal direction, as Fig. 3 shown. For each point, the gray level profile $\mathbf{g}_{j}^{i}$ is computed. The retained candidate point is this with the lowest Mahalanobis distance using variance $\mathbf{S}_{j}$ :

$$
f\left(\mathbf{g}_{j}^{i}\right)=\left(\mathbf{g}_{j}^{i}-\overline{\mathbf{g}_{j}}\right)^{T} \mathbf{S}_{j}^{-1}\left(\mathbf{g}_{j}^{i}-\overline{\mathbf{g}_{j}}\right)
$$

2. The displacements $d \mathbf{X}$ of the current feature points are determined from the Mahalanobis distance. The deformation component $d \mathbf{b}$ is estimated by:

$$
d \mathbf{b}=\mathbf{P}^{T} d \mathbf{X}
$$

And the shape updates as:

$$
\mathbf{x}^{\text {new }}=\overline{\mathbf{x}}+\mathbf{P} d \mathbf{b}
$$

The final point positions of the fitted model are obtained until dis $\left(\mathbf{x}^{n e w}\right.$ $\mathbf{x})<\eta$, where dis is the Euclidean distance between two vectors and $\eta$ is a very small number. Considering the perspective of fitting accuracy, we chose $\eta=0.0001$. 


\subsubsection{Active shape model for ultrasound image segmentation}

ASM has proven to be effective on several medical image segmentation, such as CT, fMRI, but it has some challenges when comes to ultrasound images segmentation. This is mainly due to the speckle nature of the ultrasound images. In an ultrasound image, one tissue in the ultrasound image appears as a homogeneous region with similar speckle distribution. The main idea of our framework is: (1) to estimate an ultrasound-based image representation in which homogeneous region is characterized based on speckle specificities and (2) to use this ultrasound-based image representation as the input for ASM.

\section{$2.2 \quad$ Ultrasound Image Representation}

\subsubsection{Rayleigh Mixture Model}

This section aims at providing speckle property from an RMM description of the ultrasound image. In ultrasound images, the intensity distributions have been demonstrated to be Rayleigh distributions due to the speckle [22, $24,25]$. The histogram of ultrasound Region of Interest (ROI) is shown in Fig. 4. Fig. 4(a) shows the ROI inside the yellow bound box while Fig. 4(b) presents the histogram of ROI that is more likely a Rayleigh distribution.

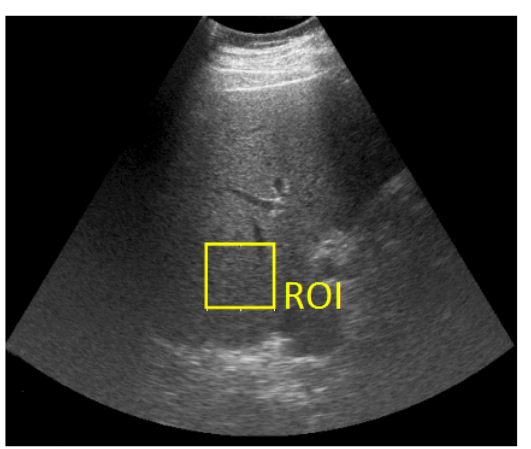

(a)

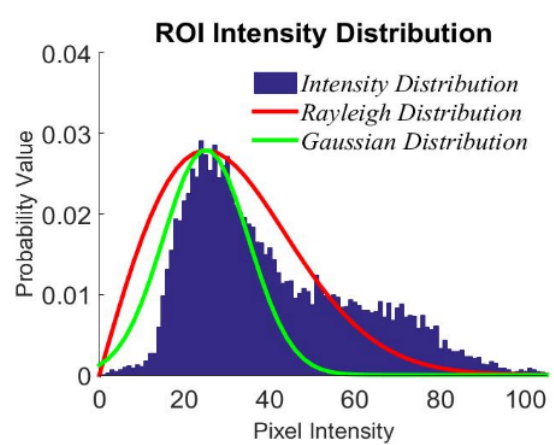

(b)

Figure 4: The liver part of the abdominal ultrasound image as the region of interest (a) and the histogram for the ROI (b).

Let $\mathbf{Z}=\left\{z_{1}, z-2, \ldots, z_{N}\right\}$ be a set of pixel intensities of a given region of interest of $N$ pixels from an ultrasound image. We assume that the pixel intensities can be described by the following mixture of $L$ Rayleigh distributions:

$$
p\left(x_{i} \mid \Psi\right)=\sum_{j=1}^{L} \pi_{j} p\left(z_{i} \mid \sigma_{j}\right)
$$


where $L$ is the number of the Rayleigh distributions of the RMM, $\pi_{j}$ is the weight of each component, $p\left(z_{i} \mid \sigma_{j}\right)$ is the probability density function (PDF) of the Rayleigh distribution, and $\sigma_{j}$ is the variance of the Rayleigh distribution. $p\left(z_{i} \mid \sigma_{j}\right)$ is given by:

$$
p\left(z_{i} \mid \sigma_{j}\right)=\frac{z_{i}}{\sigma_{j}^{2}} \exp \left(-\frac{z_{i}^{2}}{2 \sigma_{j}^{2}}\right)
$$

Eq. (8) is characterized by the parameter set $\Psi=\left\{\pi_{1}, \pi_{2}, \cdots, \pi_{L}, \sigma_{1}, \sigma_{2}, \cdots, \sigma_{L}\right\}$ composed by the weights and Rayleigh parameters of the mixture model. To guarantee that $p\left(z_{i} \mid \Psi\right)$ is a true distribution function, the condition $\sum_{j=1}^{L} \pi_{j}=1$ must hold.

The model of the whole ultrasound image is then:

$$
p(\mathbf{Z}, \Psi)=\prod_{i}^{N} p\left(z_{i} \mid \Psi\right)
$$

\subsubsection{Expectation Maximum (EM) Algorithm}

The expectation maximization (EM) algorithm is applied for parameters estimation. In RMM, the estimation of the parameter set $\Psi=\left\{\pi_{1}, \pi_{2}, \cdots, \pi_{L}, \sigma_{1}, \sigma_{2}, \cdots, \sigma_{L}\right\}$ is achieved by maximizing the likelihood function:

$$
L(\mathbf{Z}, \Psi)=\log p(Z \mid \Psi)=\sum_{i=1}^{N} \log \sum_{j=1}^{L} \pi_{j} p\left(z_{i} \mid \Psi\right)
$$

The parameters set $\hat{\Psi}$ can then be estimated by:

$$
\hat{\Psi}=\arg \max _{\Psi} L(Z, \Psi)
$$

To solve Eq. (12), the EM algorithm is applied [22]. The procedure is shown as follows.

Step 1: Initialize parameter set $\Psi^{(0)}$, where $\pi_{j}^{(0)}=\frac{1}{L}, j=\{1,2, \ldots, L\}$ and $\sigma_{j}^{(0)}=$ randomvalue;

Step 2: Expectation step (E-step). The expectation of the likelihood function is computed by:

$$
\begin{aligned}
Q^{(t)}\left(\mathbf{Z}, \Psi^{(t)}, \Psi\right) & \left.=E\left[L\left(Z, \Psi^{(t)}, \Psi\right)\right)\right] \\
& =\sum_{i=1}^{N} \sum_{j=1}^{L} \gamma_{i j}\left[\log p\left(z_{i}, \sigma_{j}\right)+\log \pi_{j}\right]
\end{aligned}
$$


where $\Psi^{(t)}$ is the previous estimation of the parameters and $\gamma_{i j}$ is the distribution of the unobserved variables, which is defined as follows:

$$
\gamma_{i j}=\frac{\pi_{i j} p\left(z_{i} \mid \sigma_{j}\right)}{p\left(z_{i} \mid \Psi^{(t)}\right)}
$$

Step 3: Maximization step (M-step). The new parameter set $\Psi^{(t+1)}$ is achieved by:

$$
\begin{gathered}
\Psi^{(t+1)}=\arg \max _{\Psi} Q^{(t)}\left(\mathbf{Z}, \Psi^{(t)}, \Psi\right) \\
\pi_{j}^{(t+1)}=\frac{1}{N} \sum_{i=1}^{N} \gamma_{i j}^{(t)} \\
\sigma_{j}^{(t)}=\sqrt{\frac{1}{N} \sum_{i=1}^{N} \gamma_{i j}^{(t)} \frac{z_{i}^{2}}{2}}
\end{gathered}
$$

When the EM algorithm procedure is finished, the parameters of RMM are obtained. Once the RMM is established, the clustering images are obtained from the original images. Fig. 5 shows the generation of clustering images from original images with three examples.

\section{Active shape model with Rayleigh features}

ASM-RMMC method is proposed for the prostate ultrasound image segmentation. Similar to conventional ASM, ASM-RMMC consists of training stage and testing stage as shown in Fig. 6. In this flowchart, the blue part shows the procedure of the training stage while the red part shows the testing stage.

Training stage: In order to achieve the mean shape and the main variation modes described in Eq. (1) - Eq. (3), the statistical shape model is built from the landmarks as described in Section 2.1. Then, the clustering images are generated through RMM as described in Section 2.2 and used to build the appearance model as described in Section 2.1.

Testing stage: In order to delineate the prostate on unlabeled ultrasound images, a two-steps approach is performed as follows.

Step 1: Ultrasound representative image generation. The RMM is used directly to generate the clustering images to take part in the deformation stage. 
Original Ultrasound Images

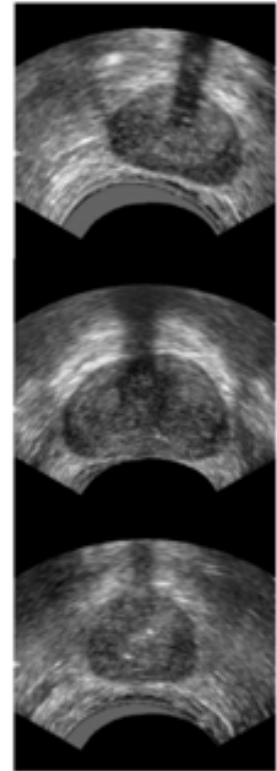

Rayleigh Mixture Model

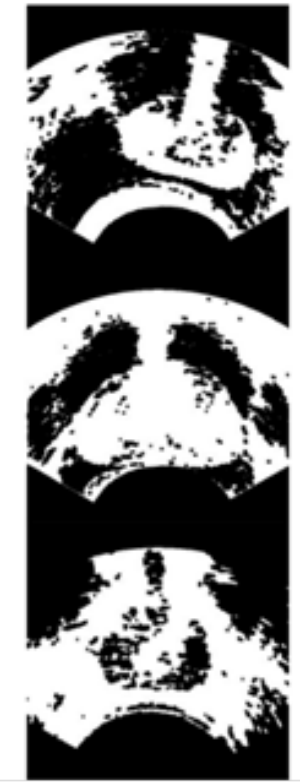

Figure 5: The generation of clustering images from original images using Rayleigh Mixture Model.

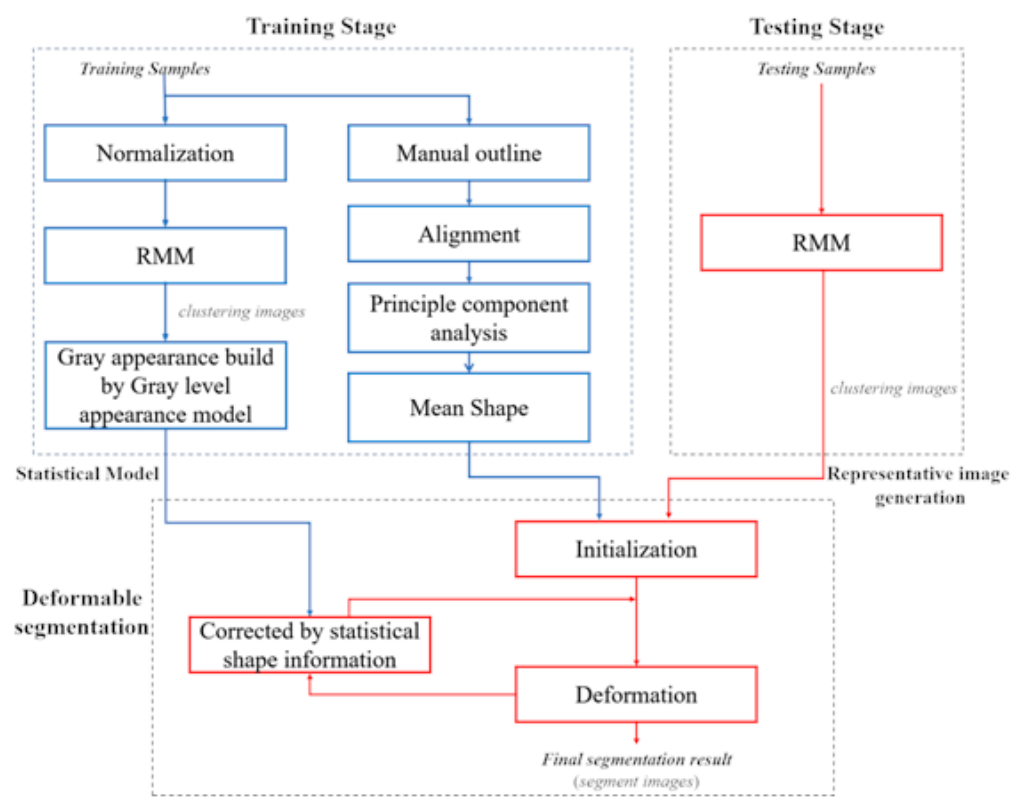

Figure 6: The flowchart of Active Shape Model with Rayleigh Mixture Model clustering method including training stage (shown in blue) and testing stage (shown in red). 
Step 2: Deformation. The trained mean shape on the clustering images as initialization. The shape is iterative deformed according to the approach described in Eq. (5) - Eq. (7) until the convergence condition holds.

\section{Experiments}

In this section, experiments were conducted to evaluate the proposed method on in vivo prostate ultrasound images. The proposed method was implemented in MATLAB R2019a on an Intel(R) Core(TM) i5 6500 computer, $3.40 \mathrm{GHz}$ and $8 \mathrm{~GB}$ RAM.

\subsection{Ultrasound images dataset}

The ultrasound data are transrectal prostate ultrasound (TRUS) image acquired intra-operatively on a prostate HIFU therapy Ablatherm device (https://www.edap-tms.com/) on real patients receiving prostate HIFU ablation. The data were provided and anonymized directly by EDAP company, (Vaulx-en-Velin, France). They were acquired with the patients has written consent under the clinical condition at Edouard Herriot Hospital, Lyon, France. Moreover, this study was approved by the Institutional Review Board of Edouard Herriot Hospital. On these patients, a urethral catheter was placed to protect the urethra from injury during HIFU ablation, but this catheter-induced an ultrasonic shadow as it shown in Fig. 5(a). The transrectal ultrasound imaging probe is operating at $7.5 \mathrm{MHz}$. A slice has $500 \times 490$ pixels with a transverse pixel size of $0.154 \mathrm{~mm} /$ pixel and a thickness of $2 \mathrm{~mm}$.

\subsection{Qualitative evaluation}

At first, we evaluated the proposed method from the visual quality point of view. The procedure of the proposed method consisted of two stages: (1) Initialization and (2) ASM deformation on the clustering images as shown in Fig. 6. Fig. 7 shows two representative segmentation of ASM-RMMC. The first and second rows show the deformations from patient \#1 and patient $\# 2$, respectively. The initialization is shown in Fig. 7 column (a). The deformations are illustrated in Fig. 7 columns (b) - (d) which present the intermediate shapes after 5, 10, 15 iteration steps. When the deformations satisfied $\operatorname{dis}\left(\mathbf{x}^{\text {new }}-\mathbf{x}\right)<0.0001$, the final results are achieved, as shown in Fig. 7 column (e).

We also compared the visual quality between ASM-RMMC and two other close methods in which the clustering image is computed using different methods. One is ASM-GFC that utilizes Gabor features for the clustering image while the other is ASM-GMMC that utilizes the classical Gaus- 


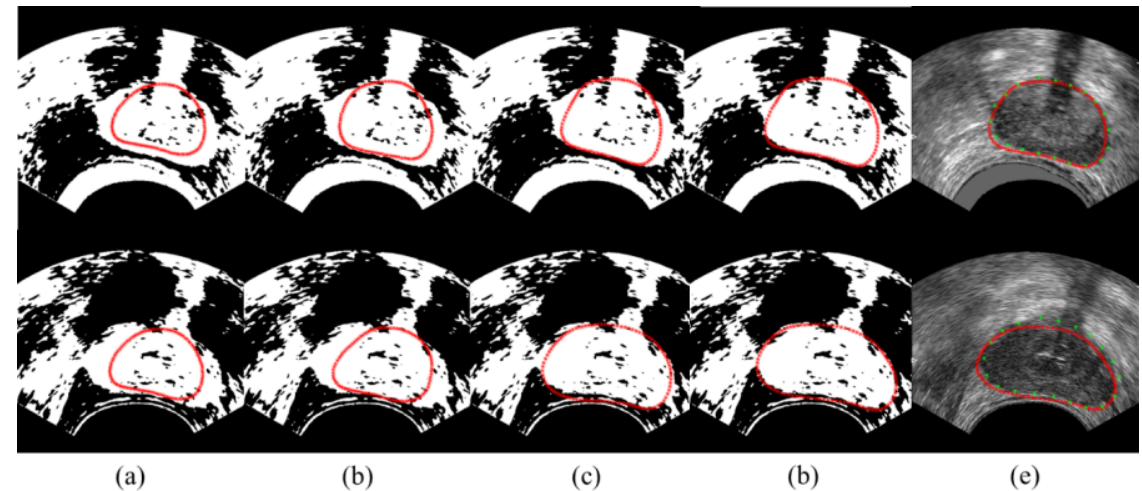

Figure 7: The results of prostate segmentation by using Active Shape Model with Rayleigh Mixture Model clustering method for two patients.

sian Mixture Model (GMM). Gabor filters is a conventional texture feature extraction tool and has been applied for prostate ultrasound images segmentation using ASM in [11]. Fig. 8 shows the Gabor features clustering process. The original images are shown in Fig. 8(a). Firstly, Gabor features (6 scales, 12 directions) are extracted and then the K-means algorithm is used to generate the clustering images as shown in Fig. 8(b). GMM is the most common finite model that many previous works are based on it for images segmentation [20]. Similar to the framework described in section 2.2, the parameters of the GMM were estimated using the EM algorithm and then the GMM was used to produce a clustering image.

Fig. 9 presents the segmentation results on five images of the testing dataset obtained by ASM-GFC, ASM-GMMC, and ASM-RMMC. The red dots indicate contours estimated by the segmentation algorithms while the star lines indicate manual delineations by an expert. The segmentation results provided by ASM-GFC, ASM-GMMC, and ASM-RMMC are displayed on the first, second, and third column, respectively. The first to fifth lines in Fig. 9 present the segmentation results of five patients. Obviously, ASMRMMC is the most effective to detect prostate contours from ultrasound images in visually. The reason is that the clustering images generated by RMM clustering further reduce inhomogeneity of ultrasound image.

\subsection{Quantitative evaluation}

In order to evaluate the proposed method quantitatively, we also compared ASM-RMMC with ASM-GFC and ASM-GMMC. Four evaluation indexes were applied: Dice Similarity Coefficient (DSC), Mean Average Distance (MAD), False Positive Error (FPE) and False Negative Error (FNE) [28].

DSC is used to measure the overlap between the areas of the segmented contour $\left(S_{s}\right)$ to this of the manual delineation $\left(S_{d}\right)$ considered as ground- 


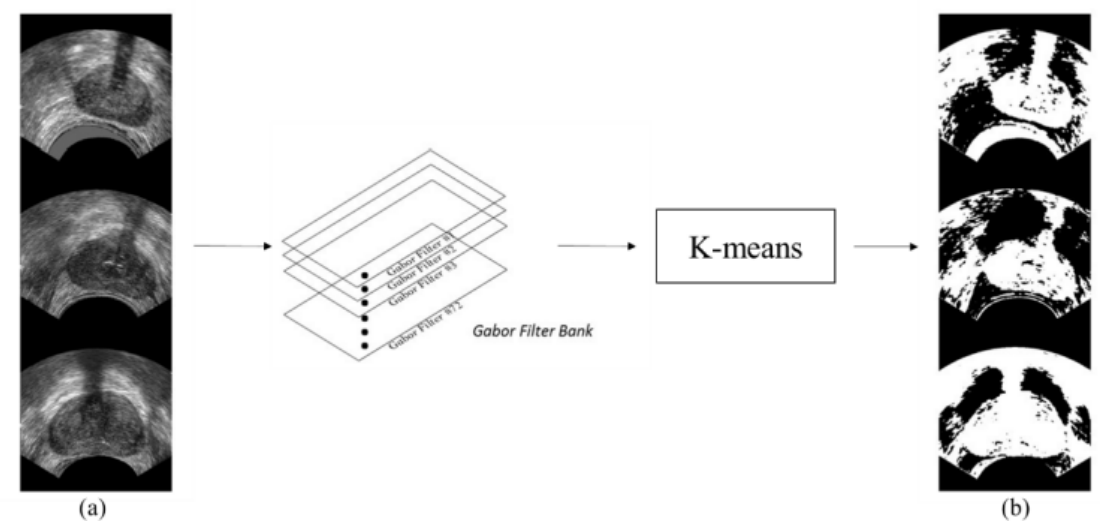

Figure 8: The illustration of the Gabor features clustering from the original images (a) to the clustering images (b).

truth [29]. The DSC gives a value between 1 (perfect overlap) to 0 (no overlap).

$$
D S C=\frac{2\left(S_{s} \cap S_{d}\right)}{\left(S_{s}\right)+\left(S_{d}\right)}
$$

The MAD is a distance-based metric that calculates the average (in pixels) of the absolute closest distances expressed between the estimated contour and each point of the manually delineated landmarks. The closer MAD is to 0, the better is the segmentation result.

FPE (Eq. (19)) is defined as the ratio of the number of the total pixels of the segmented prostate region outside the ground-truth (the false positives) to the number of the total pixels of the ground-truth $[28,30,31]$. FNE (Eq. (20)) is defined as the ratio of the number of the total pixels of the ground-truth pixels outside the segmented prostate region (the false negatives) to the number of the total pixels of the ground-truth [28, 30, 31].

$$
\begin{aligned}
& F P E=\frac{S_{s}-\left(S_{s} \cap S_{d}\right)}{S_{d}} \times 100 \% \\
& F P E=\frac{S_{d}-\left(S_{s} \cap S_{d}\right)}{S_{d}} \times 100 \%
\end{aligned}
$$

Considering the small size of the dataset (30 ultrasound images), we utilized 6-fold cross-validation to evaluate the proposed method. The 6fold cross-validation separated the dataset into 6 parts randomly, and we utilized one part as test images. That means, 25 of these images are selected randomly as the training set while the other 5 prostate ultrasound images are used as the testing set. Six experiments were conducted and the results are shown in Table 1. 


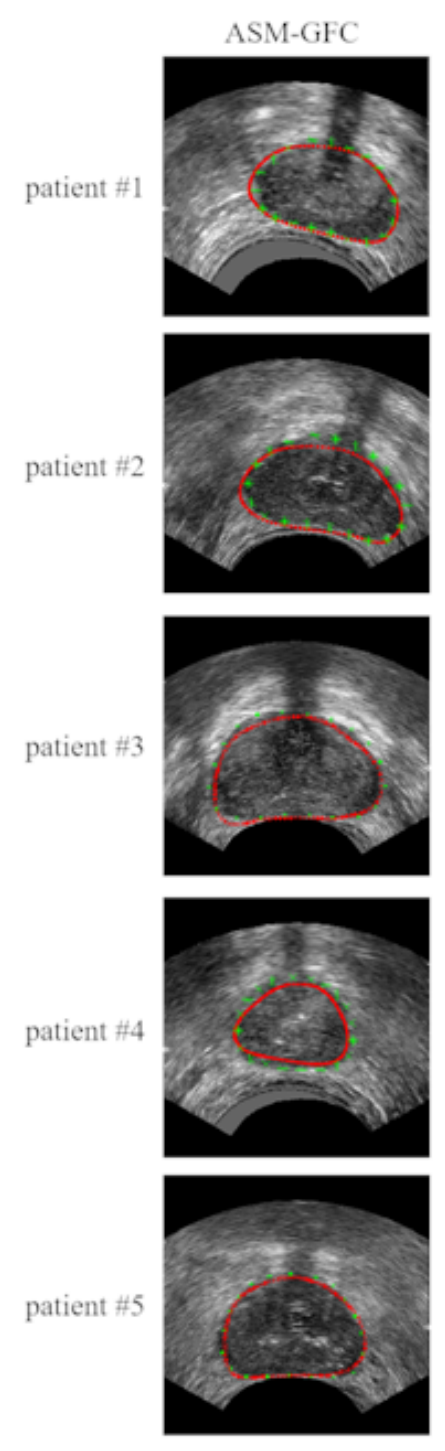

(a)

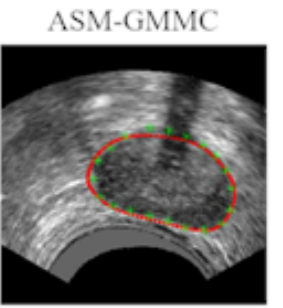

ASM-RMMC
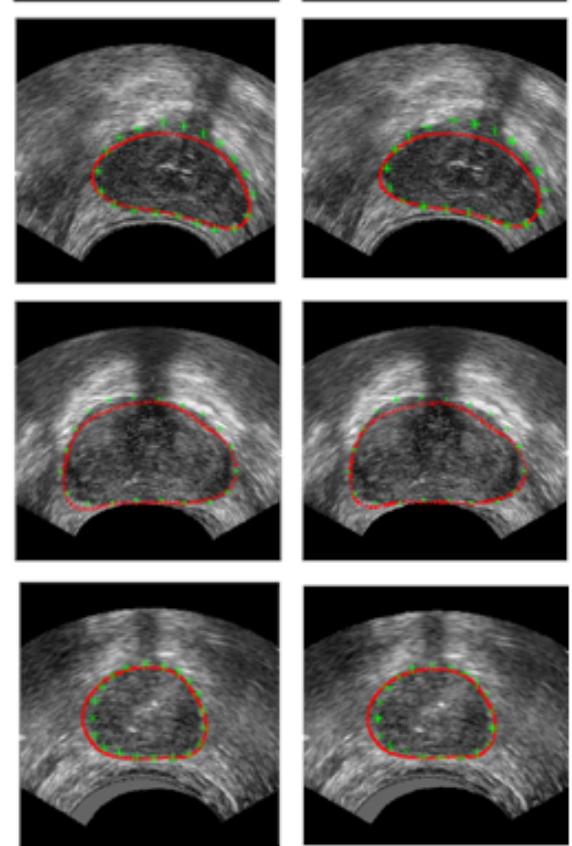

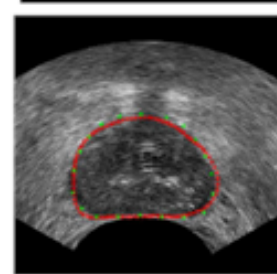

(b)

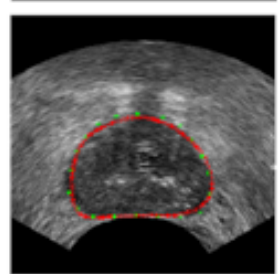

(c)

Figure 9: The results of prostate ultrasound image segmentation for five patients $(\# 1-\# 5)$. 
DSC aspect:As shown in Table 1, ASM-RMMC reached scores of 95\% \pm $0.81 \%$ which is an improvement of $2 \%$ compare to the scores obtained by ASM-GFC $(93 \% \pm 1.47 \%)$. We can also see that ASM-RMMC has the same mean DSC scores as ASM-GMMC $(95 \% \pm 1.17 \%)$, but its standard deviation is slightly smaller that means the ASM-RMMC is more robust. MAD aspect: ASM-RMMC reached MAD of $1.86 \pm 0.02$ pixels while ASM-GFC achieved MAD of $2.29 \pm 0.04$ pixels and ASM-GMMC achieved $1.92 \pm 0.03$ pixels. Clearly, compared to ASM-GFC and ASM-GMMC, the MAD obtained by ASM-RMMC decreases by $18.78 \%$ and $3.13 \%$, respectively. These decreases indicated that ASM-RMMC estimated more accurately the contours than ASM-GFC and ASM-GMMC. FPE aspect: the FPE of ASM-GFC, ASMGMMC and ASM-RMMC are $3.67 \% \pm 0.74 \%, 2.88 \% \pm 0.52 \%$, and $2.10 \% \pm$ $0.36 \%$, respectively. FNE aspect: the FNE of ASM-GFC, ASM-GMMC and ASM-RMMC are $4.24 \% \pm 0.47 \%, 3.39 \% \pm 0.49 \%$, and $2.78 \% \pm 0.71 \%$, respectively. In both FPE and FNE metrics, ASM-RMMC showed better scores than ASM-GFC and ASM-GMMC.

The improvement of the scores can also be noticed on the boxplots in Fig. 10. For all the metrics ASM-RMMC shows better median values and smaller inter-quartiles ranges than ASM-GFC and ASM-GMMC. The statistics show that the proposed method ASM-RMMC outperforms ASM-GFC and ASM-GMMC. The Kruskal-Wallis test by rank (the non-parametric equivalent of ANOVA) was conducted to find if significant differences exist between the scores distributions of ASM-GFC, ASM-GMMC and ASMRMMC. The respective $p$ values $(p<0.05$ for DSC, $p<0.001$ for MAD, $p<0.005$ for FPE and $p<0.05$ for FNE) allowed us to conclude that the accuracy improvements brought by ASM-RMMC are statistically significant.

\begin{tabular}{cccccccccc}
\hline & & Test1 & Test2 & Test3 & Test4 & Test5 & Test6 & mean & std dev \\
\hline Mean & ASM-GFC & 94 & 92 & 93 & 94 & 95 & 91 & 93 & 1.47 \\
ASC & ASM-GMMC & 95 & 93 & 96 & 95 & 96 & 94 & 95 & 1.17 \\
$\%$ & ASM-RMMC & $\mathbf{9 5}$ & $\mathbf{9 4}$ & $\mathbf{9 6}$ & $\mathbf{9 6}$ & $\mathbf{9 6}$ & $\mathbf{9 5}$ & $\mathbf{9 5}$ & $\mathbf{0 . 8 1}$ \\
\hline \multirow{2}{*}{ Mean } & ASM-GFC & 2.27 & 2.32 & 2.29 & 2.27 & 2.24 & 2.34 & 2.29 & 0.04 \\
pixel & ASM-GMMC & 1.91 & 1.97 & 1.95 & 1.92 & 1.89 & 1.93 & 1.92 & 0.03 \\
\hline \multirow{2}{*}{ ASM-RMM } & ASM-GFC & $\mathbf{1 . 8 7}$ & $\mathbf{1 . 8 9}$ & $\mathbf{1 . 8 5}$ & $\mathbf{1 . 8 4}$ & $\mathbf{1 . 8 5}$ & $\mathbf{1 . 8 8}$ & $\mathbf{1 . 8 6}$ & $\mathbf{0 . 0 2}$ \\
\% & ASM-GMMC & 2.76 & 2.98 & 4.05 & 4.67 & 2.64 & 3.01 & 3.67 & 0.74 \\
\hline \multirow{2}{*}{ Mean } & ASM-RMMC & $\mathbf{2 . 1 4}$ & $\mathbf{2 . 1 9}$ & $\mathbf{2 . 1 7}$ & $\mathbf{2 . 6 0}$ & $\mathbf{1 . 5 8}$ & $\mathbf{1 . 7 9}$ & $\mathbf{2 . 1 0}$ & $\mathbf{0 . 3 6}$ \\
$\%$ & ASM-GFC & 4.21 & 4.46 & 4.81 & 3.39 & 4.35 & 4.21 & 4.24 & 0.47 \\
ASM-GMMC & 3.13 & 3.46 & 3.78 & 2.54 & 3.86 & 3.56 & 3.39 & 0.49 \\
& ASM-RMMC & $\mathbf{2 . 7 1}$ & $\mathbf{2 . 8 7}$ & $\mathbf{3 . 1 3}$ & $\mathbf{1 . 4 2}$ & $\mathbf{3 . 3 9}$ & $\mathbf{3 . 1 8}$ & $\mathbf{2 . 7 8}$ & $\mathbf{0 . 7 1}$ \\
\hline
\end{tabular}

Table 1: Review of segmentation performances announced in 6-fold cross validation (Mean DSC, MAD, FPE and FNE). 


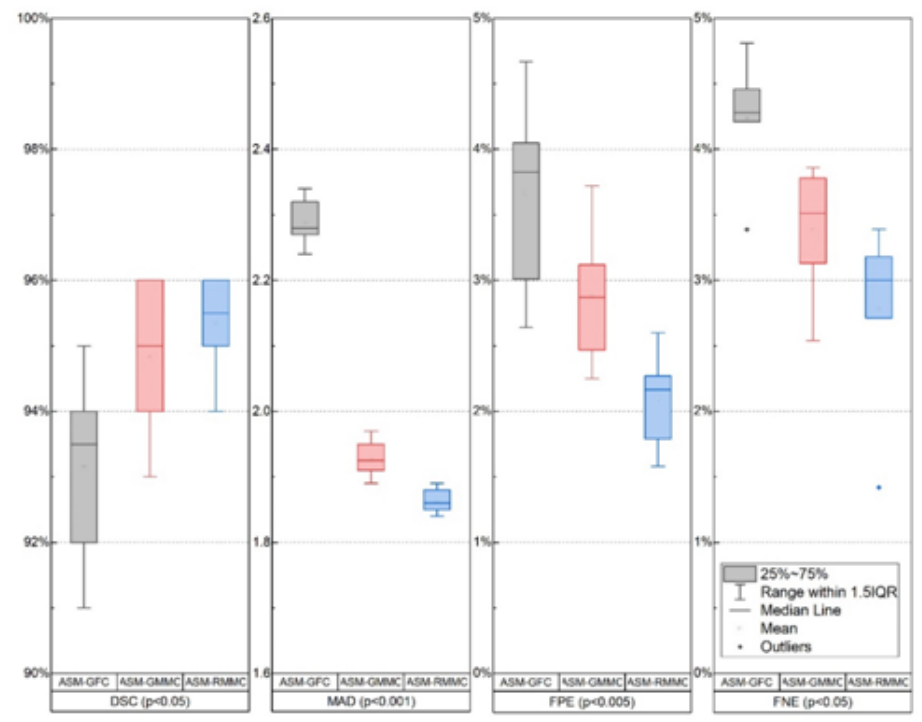

Figure 10: Boxplots of the DSC, MAD, FPE and FNE obtained by 6 -fold cross validation of ASM-GFC, ASM-GMMC and ASM-RMMC. The $p$ values were computed with the Kruskal-Wallis test by rank.

Furthermore, the obtained DSC and MAD scores of our proposed ASMRMMC is compared with these mentioned in four related works of the literature: Ladak [32, 33], Cosio [34], Yan [35] and Ghose [36] (see Table 2). In these papers, there are two kind of units to represent the results of the MAD: as pixels and as mm. Compared to Ladak and Ghose, the ASM-RMMC MAD is decreased from $4.4 \pm 1.8 / 4.5 \pm 2.14$ pixels to $1.87 \pm 0.51$ pixels by $57 \%$ and $58 \%$, respectively. Compared to the works of Cosio, Yan, and Ghose, the ASM-RMMC MAD is decreased from $1.65 \pm 0.67 / 2.1 \pm 1.02 / 2.1 \pm 1.02$ $\mathrm{mm}$ to $0.28 \pm 0.15 \mathrm{~mm}$ by $63 \%, 66 \%$, and $57 \%$, respectively. Moreover, compared to Ghose, the DSC of ASM-RMMC is increased from $91 \% \pm 9 \%$ to $95 \% \pm 1 \%$.

The computation time of ASM-GFC, ASM-GMMC, and ASM-RMMC is shown in Table 3 and Fig. 11. The mean total time for ASM-GFC reaches $780.01 \mathrm{~s}$, in which about $729 \mathrm{~s}$ are spent for Gabor features extraction, 46.53 $\mathrm{s}$ for clustering and $3.06 \mathrm{~s}$ for ASM. The mean total time for ASM-GMMC takes $42.49 \mathrm{~s}$ (merely 1/18 of ASM-GFC's time cost), in which about 39.26 $\mathrm{s}$ is used for GMM and $3.23 \mathrm{~s}$ for ASM. Notably, the mean total time for ASM-RMMC is less than $8 \mathrm{~s}$ (merely 1/98 of ASM-GFC's time cost), in which about $4.91 \mathrm{~s}$ is used for RMM and $2.98 \mathrm{~s}$ for ASM. The proposed ASM-RMMC is the most efficient. The reason is that RMM costs much less computation time during ultrasound image clustering since it has fewer parameters to be estimated than GMM. 


\begin{tabular}{lcc}
\hline Method & MAD & DSC \\
\hline Ladak [32,33] & $4.4 \pm 1.8$ pixels / - & - \\
Cosio $[34]$ & $-/ 1.65 \pm 0.67 \mathrm{~mm}$ & - \\
Yan $[35]$ & $-/ 2.1 \pm 1.02 \mathrm{~mm}$ & - \\
Ghose $[36]$ & $4.5 \pm 2.14$ pixels $/ 1.26 \pm 0.6 \mathrm{~mm}$ & $91 \pm 9$ \\
ASM-RMMC & $1.87 \pm 0.51$ pixels / $0.28 \pm 0.15 \mathrm{~mm}$ & $95 \pm 1$ \\
\hline
\end{tabular}

Table 2: Review of segmentation performances announced in the literature (MAD and DSC).

\begin{tabular}{cccccccc}
\hline Method & Stage & $\# 1$ & $\# 2$ & $\# 3$ & $\# 4$ & $\# 5$ & mean \\
\hline \multirow{3}{*}{ ASM-GFC } & Clustering & 749.45 & 773.15 & 785.66 & 773.59 & 791.89 & 776.75 \\
& ASM & 3.69 & 3.84 & 3.92 & 1.20 & 3.65 & 3.06 \\
& Total & 763.14 & 776.99 & 789.58 & 774.79 & 795.54 & 780.01 \\
\hline \multirow{3}{*}{ ASM-GMMC } & Clustering & 37.42 & 43.23 & 35.60 & 41.91 & 38.12 & 39.26 \\
& ASM & 3.72 & 3.81 & 3.86 & 1.20 & 3.56 & 3.23 \\
& Total & 41.14 & 47.04 & 39.46 & 43.11 & 41.68 & 42.49 \\
\hline \multirow{3}{*}{ ASM-RMMC } & Clustering & 4.81 & 4.54 & 4.56 & 4.81 & 4.70 & 4.91 \\
& ASM & 3.78 & 3.79 & 3.76 & 1.17 & 3.51 & 2.97 \\
& Total & 8.59 & 8.33 & 8.32 & 5.98 & 8.21 & 7.88 \\
\hline
\end{tabular}

Table 3: Computation time of segmentation on five testing patients' data (\#1-5) using ASM-GFC, ASM-GMMC, and ASM-RMMC. (Unit: seconds)

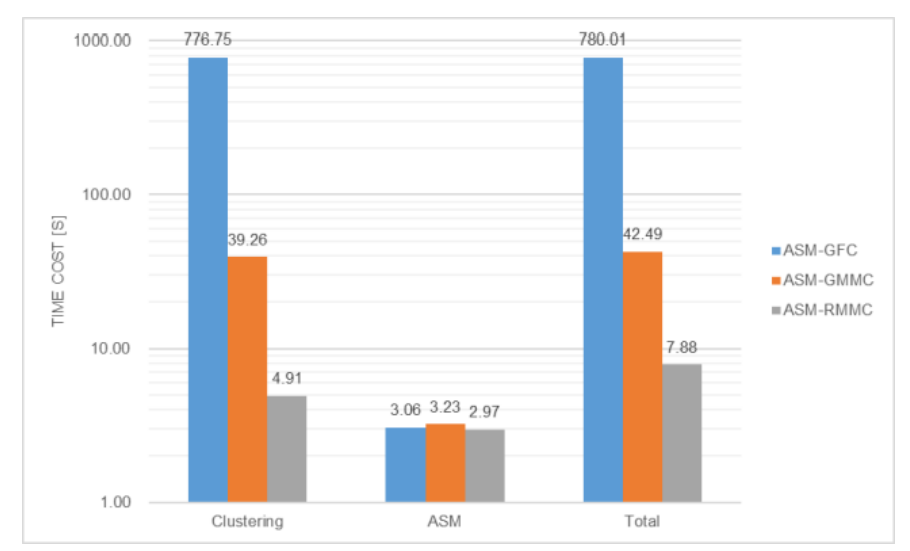

Figure 11: Computation time of segmentation using ASM-GFC, ASMGMMC, and ASM-RMMC. 


\section{Discussion}

Active shape model (ASM) is a method which generally gives good methods since it is able to catch relatively large shape variability. However, when directly applied on US images, the estimation of the shape deformations is difficult because of the gray level in-homogeneities induced by the speckle. This problem can be by-passed by extracting some region-based information from statistics on speckle and using it to perform the ASM. Several statistics on the speckle have been proposed (texture features, Gabor filter, Mixture Model, ...). However our feeling was to use some statistics related to the physics of ultrasound imaging. The fully developed speckle model indicates that, in B-mode ultrasound, the pixels intensities follow a Rayleigh distribution. We considered so the US image as a Mixture of Rayleigh distributions in order to extract some more homogeneous tissues region from the US image and used this information to perform the ASM. This assumption seems to be verified since ASM-RMMC showed higher accuracy than ASM-GFC and ASM-GMMC which are based on two clustering methods, respectively on Gabor filters and on Gaussian mixture Model, which are not directly related to the physics of speckle generation.

More surprisingly, ASM-RMMC showed also higher computer time efficiency than the two other methods. This efficiency was awaited compared to ASM-GFC because the computation of the Gabor filter banks is known to be computation intensive. However, the computation of the RMM was also shorter than this of GMM because the Rayleigh distribution is based on only one parameter whereas the Gaussian distribution is defined by two parameters.

The evaluation was performed on a relative small dataset of 30 ultrasound images. However, the 6 -fold cross-correlation was able to show that ASM-RMMC had higher performances in terms of accuracy and computation time than the two other methods. This increase of performance was statistically significant since that the Kruskal-Wallis test by rank showed $p$ values always lower than $5 \%$.

However, in the future, we will endeavor to collect more prostate ultrasound images for a more deeper evaluation of the proposed ASM-RMMC and also we will attempt to extend the proposed ASM-RMMC to the US images segmentation of other organs, such as uterus, liver, ...

\section{Conclusion}

In this paper, for prostate ultrasound image segmentation, we proposed the ASM-RMMC by combining ultrasound image properties represented by Rayleigh Mixture Model (RMM) and prostate shape information represented by Active Shape Model (ASM). A clustering image with more homogeneous 
tissues regions is generated based on RMM, then the deformation of ASM is implemented on this image. We conducted some experiments on clinical prostate ultrasound images. The proposed method presents more accuracy for the prostate boundaries estimation and less computational complexity than other related works. In the future, the proposed method will be applied on other kinds of tissue that has a regular shape, such as liver and kidney.

\section{Acknowledgments}

This work was supported by the by the National Natural Science Foundation of China under Grants 61271312, 61201344, 61401085, 31571001, 81530060, by Natural Science Foundation of Jiangsu Province under Grant BK2012329, BK2012743, BK20150647, DZXX-031, BY2014127-11, by the '333' project under Grant BRA2015288, by the Qing Lan Project, and by the Key Projects of Jiangsu University Students Innovation and Entrepreneurship Training Program (201811055003Z).

\section{References}

[1] American cancer society, https://www.cancer.org/cancer/prostate-cancer/about/key-st

[2] D. C. Beyer, Permanent brachytherapy as salvage treatment for recurrent prostate cancer., Urology 54 (5) (1999) 880-883. doi:10.1016/s0090-4295(99)00241-1.

[3] C. Garnier, J.-J. Bellanger, K. Wu, H. Shu, N. Costet, R. Mathieu, R. de Crevoisier, J.-L. Coatrieux, Prostate segmentation in HIFU therapy., IEEE Transactions on Medical Imaging 30 (2011) 792-803. doi:10.1109/TMI.2010.2095465.

[4] K. K. Hodge, J. E. McNeal, M. K. Terris, T. A. Stamey, Random systematic versus directed ultrasound guided transrectal core biopsies of the prostate., The Journal of urology 142 (1989) 71-4; discussion 74-5. doi:10.1016/s0022-5347(17)38664-0.

[5] J. A. Noble, Ultrasound image segmentation and tissue characterization, Proceedings of the Institution of Mechanical Engineers, Part H: Journal of Engineering in Medicine 224 (2) (2010) 307-316.

[6] J. A. Noble, D. Boukerroui, Ultrasound image segmentation: a survey, IEEE Transactions on Medical Imaging 25 (8) (2006) 987-1010. doi:10.1109/tmi.2006.877092.

[7] R. Aarnink, R. Giesen, A. Huynen, J. De La Rosette, F. Debruyne, H. Wijkstra, A practical clinical method for contour determination in 
ultrasonographic prostate images, Ultrasound in Medicine \& Biology 20 (8) (1994) 705-717. doi:10.1016/0301-5629(94)90028-0.

[8] W. D. Richard, C. G. Keen, Automated texture-based segmentation of ultrasound images of the prostate, Computerized Medical Imaging and Graphics 20 (3) (1996) 131-140. doi:10.1016/0895-6111(96)00048-1.

[9] M. M. Choy, J. S. Jin, Morphological image analysis of left-ventricular endocardial borders in 2D echocardiograms, in: SPIE Proceedings on Medical Imaging, Vol. 2710, Newport Beach, 1996, pp. 852-863.

[10] L. Gong, S. D. Pathak, D. R. Haynor, P. S. Cho, Y. Kim, Parametric shape modeling using deformable superellipses for prostate segmentation, IEEE Transactions on Medical Imaging 23 (3) (2004) 340-349. doi:10.1109/tmi.2004.824237.

[11] D. Shen, Y. Zhan, C. Davatzikos, Segmentation of prostate boundaries from ultrasound images using statistical shape model, IEEE Transactions on Medical Imaging 22 (4) (2003) 539-551. doi:10.1109/tmi.2003.809057.

[12] T. F. Cootes, C. J. Taylor, Active shape models-'smart snakes'., in: BMVC, Vol. 92, 1992, pp. 266-275. doi:10.5244/c.6.28.

[13] T. F. Cootes, A. Hill, C. J. Taylor, J. Haslam, Use of active shape models for locating structures in medical images, Image and Vision Computing 12 (6) (1994) 355-365. doi:10.1016/0262-8856(94)90060-4.

[14] A. Skalski, A. Kos, T. Zielinski, P. Kedzierawski, P. Kukolowicz, Prostate segmentation in CT data using active shape model built by HoG and non-rigid Iterative Closest Point registration, in: 2015 IEEE International Conference on Imaging Systems and Techniques (IST), 2015, pp. 1-5. doi:10.1109/ist.2015.7294520.

[15] Q. Zhang, A. Bhalerao, E. Helm, C. Hutchinson, Active shape model unleashed with multi-scale local appearance, in: 2015 IEEE International Conference on Image Processing (ICIP), 2015, pp. 4664-4668. doi:10.1109/icip.2015.7351691.

[16] H. El-Rewaidy, E.-S. Ibrahim, A. S. Fahmy, Segmentation of the right ventricle in MRI images using a dual active shape model, IET Image Processing 10 (10) (2016) 717-723. doi:10.1049/iet-ipr.2016.0073.

[17] S. C. Mitchell, J. G. Bosch, B. P. Lelieveldt, R. J. Van der Geest, J. H. Reiber, M. Sonka, 3-d active appearance models: segmentation of cardiac MR and ultrasound images, IEEE Transactions on Medical Imaging 21 (9) (2002) 1167-1178. doi:10.1109/tmi.2002.804425. 
[18] C. Santiago, J. C. Nascimento, J. S. Marques, 2D segmentation using a robust active shape model with the EM algorithm, IEEE Transactions on Image Processing 24 (8) (2015) 2592-2601. doi:10.1109/tip.2015.2424311.

[19] C. B. Burckhardt, Speckle in ultrasound B-mode scans, IEEE Transactions on Sonics and Ultrasonics 25 (1) (1978) 1-6. doi:10.1109/tsu.1978.30978.

[20] T. Eltoft, Modeling the amplitude statistics of ultrasonic images, IEEE Transactions on Medical Imaging 25 (2) (2006) 229-240. doi:10.1109/tmi.2005.862664.

[21] E. Brusseau, C. L. de Korte, F. Mastik, J. Schaar, A. F. van der Steen, Fully automatic luminal contour segmentation in intracoronary ultrasound imaging-a statistical approach, IEEE Transactions on Medical Imaging 23 (5) (2004) 554-566. doi:10.1109/tmi.2004.825602.

[22] J. C. Seabra, F. Ciompi, O. Pujol, J. Mauri, P. Radeva, J. Sanches, Rayleigh mixture model for plaque characterization in intravascular ultrasound, IEEE Transactions on Biomedical Engineering 58 (5) (2011) 1314-1324. doi:10.1109/tbme.2011.2106498.

[23] K. V. Sorensen, S. V. Andersen, Rayleigh mixture model-based hidden markov modeling and estimation of noise in noisy speech signals, IEEE Transactions on Audio, Speech, and Language Processing 15 (3) (2007) 901-917. doi:10.1109/tasl.2006.885240.

[24] M. Pereyra, N. Dobigeon, H. Batatia, J.-Y. Tourneret, Segmentation of skin lesions in 2-D and 3-D ultrasound images using a spatially coherent generalized Rayleigh mixture model, IEEE Transactions on Medical Imaging 31 (8) (2012) 1509-1520. doi:10.1109/tmi.2012.2190617.

[25] H. Bi, H. Tang, G. Yang, B. Li, H. Shu, J.-L. Dillenseger, Fast segmentation of ultrasound images by incorporating spatial information into Rayleigh Mixture Model, IET Image Processing (2017). doi:10.1049/ietipr.2017.0166.

[26] T. F. Cootes, C. J. Taylor, D. H. Cooper, J. Graham, Training models of shape from sets of examples, in: BMVC92, Springer, 1992, pp. 9-18. doi:10.1007/978-1-4471-3201-1.

[27] J. C. Gower, Generalized procrustes analysis, Psychometrika 40 (1) (1975) 33-51. doi:10.1007/bf02291478.

[28] A. Klein, J. Andersson, B. A. Ardekani, J. Ashburner, B. Avants, M.-C. Chiang, G. E. Christensen, D. L. Collins, J. Gee, P. Hellier, J. H. Song, 
M. Jenkinson, C. Lepage, D. Rueckert, P. Thompson, T. Vercauteren, R. P. Woods, J. J. Mann, R. V. Parsey, Evaluation of 14 nonlinear deformation algorithms applied to human brain MRI registration, NeuroImage 46 (3) (2009) 786-802. doi:10.1016/j.neuroimage.2008.12.037.

[29] L. R. Dice, Measures of the amount of ecologic association between species, Ecology 26 (3) (1945) pp. 297-302. doi:10.2307/1932409.

[30] X. Yang, H. C. Yu, Y. Choi, W. Lee, B. Wang, J. Yang, H. Hwang, J. H. Kim, J. Song, B. H. Cho, H. You, A hybrid semi-automatic method for liver segmentation based on level-set methods using multiple seed points, Computer Methods and Programs in Biomedicine 113 (1) (2014) 69-79. doi:10.1016/j.cmpb.2013.08.019.

[31] X. Yang, J. D. Yang, H. P. Hwang, H. C. Yu, S. Ahn, B.-W. Kim, H. You, Segmentation of liver and vessels from CT images and classification of liver segments for preoperative liver surgical planning in living donor liver transplantation, Computer Methods and Programs in Biomedicine 158 (2018) 41-52. doi:10.1016/j.cmpb.2017.12.008.

[32] H. M. Ladak, F. Mao, Y. Wang, D. B. Downey, D. A. Steinman, A. Fenster, Prostate boundary segmentation from 2D ultrasound images, Medical Physics 27 (8) (2000) 1777-1788. doi:10.1118/1.1286722.

[33] N. Hu, D. B. Downey, A. Fenster, H. M. Ladak, Prostate boundary segmentation from 3D ultrasound images, Medical Physics 30 (7) (2003) 1648-1659. doi:10.1118/1.1586267.

[34] F. A. Cosío, Automatic initialization of an active shape model of the prostate, Medical Image Analysis 12 (4) (2008) 469-483. doi:10.1016/j.media.2008.02.001.

[35] P. Yan, S. Xu, B. Turkbey, J. Kruecker, Discrete deformable model guided by partial active shape model for TRUS image segmentation, IEEE Transactions on Biomedical Engineering 57 (5) (2010) 1158-1166. doi:10.1109/tbme.2009.2037491.

[36] S. Ghose, A. Oliver, J. Mitra, R. Martí, X. Lladó, J. Freixenet, D. Sidibé, J. C. Vilanova, J. Comet, F. Meriaudeau, A supervised learning framework of statistical shape and probability priors for automatic prostate segmentation in ultrasound images, Medical Image Analysis 17 (6) (2013) 587-600. doi:10.1016/j.media.2013.04.001. 